\title{
Hypoxic Preconditioning Preserves Cardiac Contractility and Reduces Infarct Size In Vivo
}

\author{
Gerry Van der Mieren, An Van den Bergh, Ines Nevelsteen, Annelies Vanderper, Willem Flameng \\ and Paul Herijgers*
}

Department of Cardiovascular Diseases, Laboratory of Experimental Cardiac Surgery, K.U. Leuven, Leuven, Belgium

\begin{abstract}
Background: Preconditioning is a powerful endogenous mechanism to protect the heart against ischemic damage. The second window of preconditioning (SWOP) is therapeutically the most attractive, but is hard to achieve by local cardiac ischemia $24 \mathrm{hrs}$ before the index ischemia in the many mice models for cardiovascular pathology, because of excessive mortality during the two procedures or the period in between. Hypoxic preconditioning is an attractive alternative preconditioning stimulus. To date, the SWOP after hypoxic preconditioning has not been studied in mice concerning infarct size reduction and preservation of left ventricular contractility in vivo.
\end{abstract}

Aim: To determine whether transient hypoxia can induce a SWOP in vivo in mice.

Methods: Hypoxic preconditioning was induced by 5 cycles of 6 minutes of $6 \%$ oxygen in 24 -week-old wild type mice. Twenty-four hours later, a 30 minutes coronary occlusion was performed. After 1 hour of reperfusion, in vivo cardiac pressure-conductance catheterization was performed with determination of the load-dependent and load-independent parameters. Infarct size was determined by TTC-staining. Sham procedures were used to obtain non-preconditioned controls.

Results: There was no mortality with the hypoxic preconditioning protocol. The left ventricular contractile parameters ejection fraction, end-systolic elastance and preload recruitable stroke work were significantly better preserved after ischemia in the preconditioned group. Diastolic relaxation (tau) was also significantly better preserved. Infarct size was reduced to half that of the non-preconditioned group.

Conclusion: Hypoxic preconditioning is a feasible stimulus to induce in vivo a second window of preconditioning in mice. Infarct size is reduced and cardiac contractility better preserved after $30 \mathrm{~min}$ regional ischemia in vivo by hypoxic preconditioning 24 hrs earlier.

\section{INTRODUCTION}

Preconditioning describes the phenomenon that a short episode of preceding ischemia induces improved tolerance in an organ or tissue to the deleterious effects of a subsequent longer period of ischemia [1-12]. Ischemic preconditioning is a powerful endogenous mechanism to protect the heart against ischemic damage. The second window of preconditioning (SWOP) is therapeutically the most attractive with its long standing effect from 24 hours till 72 hours. This protection can therefore be planned more readily in clinical practice and even chronically induced when ischemia is anticipated in the following days, but not exactly predictable, e.g. in patients with cardiovascular disease undergoing noncardiac surgery.

In the past decade, numerous transgenic mouse models have been developed. They often serve as animal models to study cardiovascular diseases, for exemple heart failure, diabetes and cardiac hypertrophy. It is important to study preconditioning in these transgenic mice for 2 reasons: 1) it gives us the opportunity to investigate whether preconditioning can be

*Address correspondence to this author at the Department of Cardiovascular Diseases, Laboratory of Experimental Cardiac Surgery, K.U. Leuven, Provisorium I, Minderbroedersstraat 19, B-3000 Leuven, Belgium; Tel: +32 16 344260; Fax: +32 16 344616; E-mail: paul.herijgers@med.kuleuven.be induced in pathological conditions that would benefit most from its protective effects, and 2) it gives us the opportunity to further unravel the mechanism of preconditioning. Therefore a well-defined model of SWOP in mice is very welcome.

Intermittent hypoxia is an attractive method to try to induce SWOP in small animal models like mice. The method avoids cardiothoracic surgery and its inherent mortality in mouse models that are often expensive and difficult to breed. Secondly no anaesthesia, which can interfere with the preconditioning stimulus, is needed to perform hypoxic preconditioning.

In previous studies, hypoxia was shown to induce SWOP with cardiac protection against ischemic damage, but these studies all used in vitro techniques [13,14].

The aim of this study is to examine whether intermittent hypoxia is capable to induce preconditioning in mice in vivo that is active $24 \mathrm{hrs}$ later. We wanted to study infarct size and in vivo load-independent left ventricular contractility to accurately define the model and the preconditioning effect.

\section{METHODS AND MATERIALS}

\section{Animal Models}

Wild type mice with C57B16/J genetic background were purchased form Jackson Laboratories (Bar Harbour, Maine, 
USA) and housed at $22^{\circ} \mathrm{C}$ on a fixed 12 hour light-dark cycle. Groups were sex-matched and investigated at the age of 24 weeks. The investigation conforms with the Guide for the Care and Use of Laboratory Animals published by the US National Institutes of Health (NIH Publication No. 85-23, revised 1996). All experimental protocols were approved by the Institutional Animal Care Commission and Ethical Committee.

Four groups of each 6 animals were used : ischemia after preconditioning $(\mathrm{I}+\mathrm{P})$, ischemia without preconditioning $(\mathrm{I}+\mathrm{NP})$, sham procedure after preconditioning $($ sham $+\mathrm{P})$ and sham procedure without preconditioning. (sham $+\mathrm{NP}$ ).

\section{Intermittent Hypoxia}

Mice were placed in a Plexiglas container (Agnthos, Sweden) of 0.8 liter. A portable oxymeter (Analox $\mathrm{O}_{2}$ EII, North Yorkshire, UK) was calibrated at $20.7 \%$ for room air and was subsequently placed in this Plexiglas container for continuous registration of the oxygen content. A mixture of $100 \%$ nitrogen and compressed air was blown through this container via 2 separate flowmeters. Within 30 seconds, the oxygen content was lowered to $6 \%$ oxygen. Five cycles of 6 minutes of $6 \%$ oxygen, interspersed with 6 minutes of room air were used [13]. In the non-preconditioned group, mice were placed in the plexiglas container, with only compressed air (without 100\% nitrogen) blown through the container.

After this hypoxic preconditioning protocol, mice were placed in their regular cage with food and water ad libitum for 24 hours.

\section{Coronary Occlusion}

Twenty-four hours later, anesthesia was induced with urethane $(1.2 \mathrm{~g} / \mathrm{kg})$ and alfa-chloralose $(50 \mathrm{mg} / \mathrm{kg})$ intraperitoneally injected. Mice were placed on a heating pad, and rectal temperature kept between 36.5 and $37.5^{\circ} \mathrm{C}$. Surgery was performed under a surgical microscope. Through a midline neck incision a tracheostomy was performed and mechanical ventilation started with room air (Minivent 845; Hugo Sachs/Harvard Apparatus, March-Hugstetten, Germany).

A left anterolateral thoracotomy was performed in the $3^{\text {th }}$ intercostal space. The left lower limb was positioned to the right to facilitate visualization of the left coronary artery (LAD). Two millimeter below the tip of the left auricle, a $8 / 0$ polypropylene suture was passed under the LAD. The snare was tightened on a small plastic tubing piece of $1.5 \mathrm{~mm}$ in order to perform an atraumatic occlusion of the LAD. Successful coronary occlusion was visually verified by observing the distal part turning pale. Occlusion was maintained for 30 minutes. Afterwards, the snare was released, reperfusion visually checked by blushing of the area, the chest closed and a reperfusion period of 1 hour allowed before the left ventricular pressure-conductance measurements were started.

\section{Outcome Parameters: Infarct Size Determination and In Vivo Left Ventricular Contractility}

Towards the end of the $1 \mathrm{hr}$ reperfusion period, the right carotid artery and jugular vein were prepared to allow the starting of measurements after exactly $60 \mathrm{~min}$ of reperfusion. A 1.4 Fr high fidelity pressure-conductance catheter (1.4-Fr, SPR-839; Millar Instruments, Houston, TX) was inserted through the right carotid artery into the left ventricle. After stabilization of the hemodynamic situation, baseline pressure-volume (PV) loops were recorded (Powerlab/4SP ADInstruments, Castle Hill, Australia) while the ventilation was momentarily turned off to avoid respiratory fluctuation of cardiac signals.

A small laparotomy was performed in the midline of the upper abdomen. The inferior caval vein was compressed between liver and diaphragm with a cotton swab, while PVloops were recorded (occlusion loops). This was performed at least 5 times. Afterwards a $24 \mathrm{G}$ catheter was introduced in the right jugular vein and parallel volume was determined by a bolus injection of $3 \mu 1$ of $30 \%$ sodium chloride solution. This was performed 3 times. The pressure-conductance catheter was removed and the carotid artery ligated. The chest was reopened and the LAD was re-occluded with a $8 / 0$ polypropylene suture at the initial occlusion site. The laparotomy was extended and the inferior caval vein was exposed infrahepatically. With a $24 \mathrm{G}$ needle, $0.3 \mathrm{ml}$ blood was retrieved from the inferior caval vein to measure specific conductivity in 3 precalibrated cuvets.

Evans blue $(0.8 \mathrm{ml}, 1 \%$ solution $)$ was given intravenously via an exchanged catheter in the right jugular vein to determine the left ventricular perfusion area at risk. After the administration of Evans blue, there was a cardiac arrest within 30 seconds. The heart was excised and placed in tissue freezing medium (Tissue-Tek, Sakura Finetek, Torrance, USA) in a $-20^{\circ} \mathrm{C}$ freezer for at least one hour. With a custom-made razorblade system, the heart was cut in slices of 1 $\mathrm{mm}$ thickness. These slices were placed for 20 minutes in triphenyl tetrazolium chloride solution $\left(1 \%, 37^{\circ} \mathrm{C}\right.$, phosphate buffer $\mathrm{pH}$ 7.4). Afterwards they were placed for 10 minutes in paraformaldehyde $\left(4 \%\right.$ solution, $\left.20{ }^{\circ} \mathrm{C}\right)$. All slices were weighed and photographed with a digital camera under microscopic magnification.

\section{Data Management and Statistical Analysis}

Analysis of the pressure-conductance data was performed using PVAN 3.2 software (Millar Instruments, Houston, TX). A conductance-volume calibration line was constructed with the cuvette data. All data were corrected for parallel volume and expressed in absolute volumes. Only technically acceptable loops were included in the analysis for each experiment [15].

Analysis of the morphological data was performed for each experiment. The number of pixels in each zone (total slice, risk zone, infarcted zone) was determined for all the slices of each heart with Adobe Photoshop 8.0 (Adobe System Inc.). After correction for the weight of the respective slices, the risk zone and infarcted zone were calculated for the entire heart.

Data are expressed as mean \pm Standard Deviation. Differences between groups were analyzed for statistical significance by factorial ANOVA followed by a LSD post hoc test. Statistical software (Statistica 7.1, StatSoft) was used. A value of $p<0.05$ was considered significant.

\section{RESULTS}

Weight, heart weight and heart weight corrected for the tibial length were equal in the 4 groups. 


\section{Acute Effects of the Intermittent Hypoxia}

All 24 mice survived the hypoxic preconditioning procedure. After initial agitation during lowering the oxygen content in the container, the mice calmed down when the oxygen content reached $6 \%$ after 30 seconds with however persistent tachypnea. After 15 seconds of exposure to room air, they demonstrated their normal behaviour pattern.

\section{Mortality During Cardiac Ischemia and Reperfusion}

In the I + NP group, 1 mouse died during the reperfusion phase after LAD occlusion. There was no mortality in the other groups.

\section{Infarct Size (Table 1)}

In both sham groups with and without the preconditioning procedure but without cardiac ischemia, there was no detectable infarct after the experimental protocol. Second window of hypoxic preconditioning was able to reduce infarct size to half that of the non-preconditioned group (49.9 \pm $7.3 \%$ to $24.7 \pm 7.3 \%$ of the risk zone, $\mathrm{P}<0.001$ ).

\section{Left Ventricular Contractility (Table 1)}

The hypoxic preconditioning protocol in the sham groups not undergoing $30 \mathrm{~min}$ index ischemia $24 \mathrm{hrs}$ later did not change left ventricular contractility parameters.

\section{Load-Dependent Parameters}

Heart rate was comparable in all groups. The risk zone of the heart was in both ischemia groups approximately $15 \%$.

Ejection fraction, a conventional but load-dependent parameter for systolic function, was better preserved when ischemia was preceded by hypoxic preconditioning $24 \mathrm{hrs}$ earlier $(56.1 \pm 5.4 \%$ vs $48.6 \pm 5.9 \%, \mathrm{P}=0.02)$.

Table 1. Phenotypic Characteristics, Infarct Size and Hemodynamic Parameters

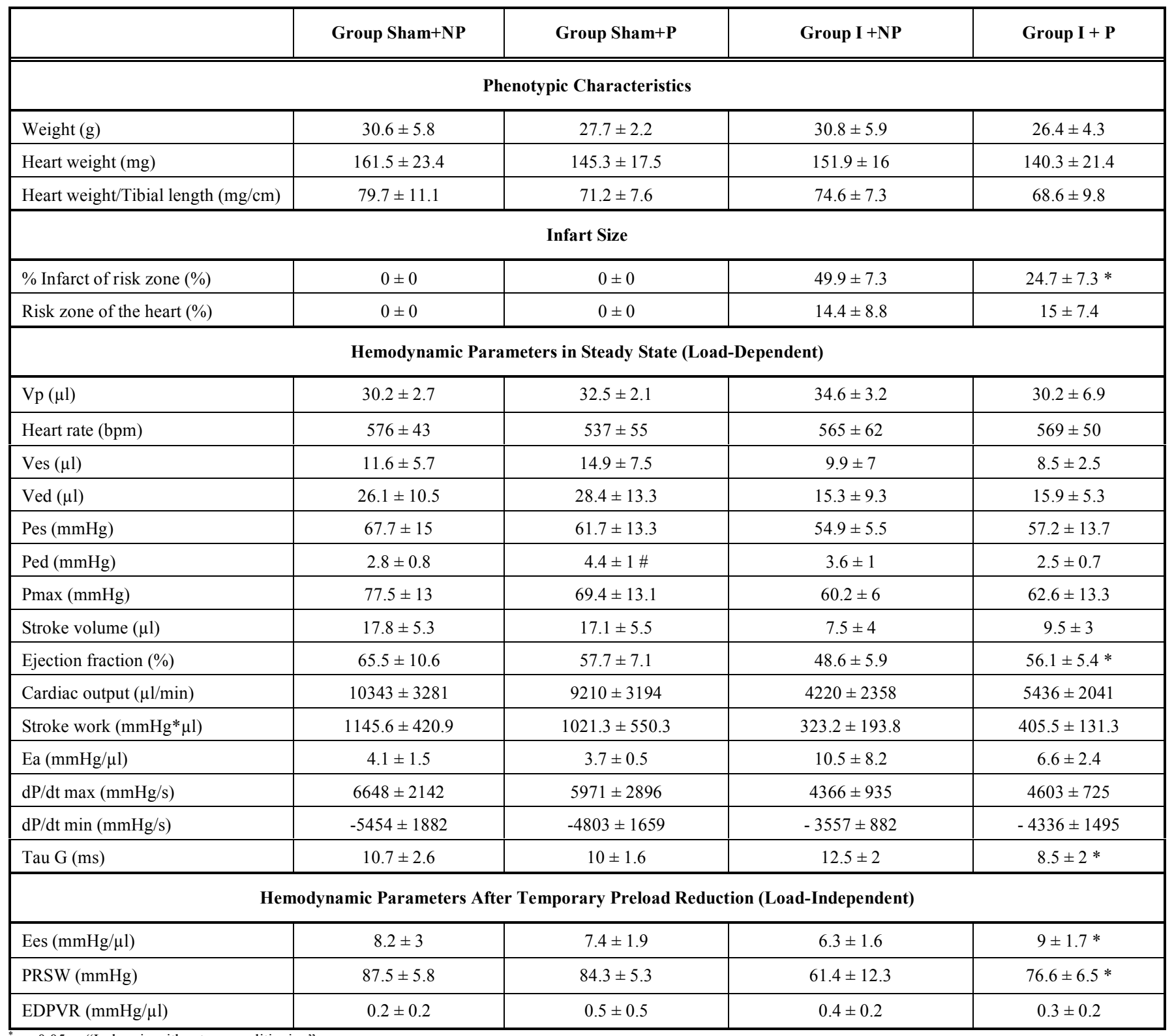

p $<0.05$ vs "Ischemia without preconditioning".

$\# \mathrm{p}<0.05$ vs "Sham procedure without preconditioning". 
The other conventional load-dependent parameters (endsystolic pressure, stroke volume, cardiac output, stroke work and $\left.\mathrm{dP} / \mathrm{dt}_{\max }\right)$ tended to be better preserved after ischemia with the preconditioning protocol, but this did not reach statistical significance.

Tau, as parameter for early diastolic relaxation was better preserved after ischemia preceded by the hypoxic preconditioning protocol $(8.5 \pm 2 \mathrm{~ms} v s 12.5 \pm 2 \mathrm{~ms}, \mathrm{P}=0.05)$. The other parameter for relaxation $\mathrm{dP} / \mathrm{dt}_{\min }$ tended to be more negative after preconditioning. No differences in left ventricular stiffness, as determined by the exponential fit of the end-diastolic pressure-volume relationship (EDPVR) could be demonstrated.

The effective arterial elastance (Ea) is defined as the ratio of the end-systolic pressure/stroke volume and is a parameter for afterload. Ea tended to be lower after preconditioning, but this didn't reach statistical significance.

\section{Load-Independent Parameters}

End-systolic elastance (Ees) is a relatively loadindependent parameter which reflects the left ventricle endsystolic stiffness and is determined as the slope of the endsystolic pressure-volume relationship. Ees was better preserved after ischemia with the preconditioning protocol $(9 \pm$ $1.7 \mathrm{mmHg} / \mu \mathrm{l}$ vs $6.3 \pm 1.6 \mathrm{mmHg} / \mu 1, \mathrm{P}=0.04$ ) (Fig. 1).

Preload Recruitable Stroke Work (PRSW) is the slope of the relationship between end-diastolic volume and stroke work performed by the ventricle. Hypoxic preconditioning $24 \mathrm{hrs}$ before 30 min regional cardiac ischemia led to a better preserved PRSW $(76.6 \pm 6.5 \mathrm{mmHg} v s 61.4 \pm 12.3 \mathrm{mmHg}$, $\mathrm{P}=0.03$ ) (Fig. 2).

\section{DISCUSSION}

Preconditioning of the heart was first described by Murry et al. in 1986 [1]. Since then, numerous papers have been published concerning this endogenous protection mechanism to unravel the underlying mechanisms [3]. In the past decade, numerous transgenic mouse models of cardiovascular diseases have been developed. The study of preconditioning in these models gives us the opportunity to investigate whether preconditioning can be induced in pathological conditions that would benefit most from its protective effects, and to further unravel the mechanism of preconditioning. Therefore, we wanted to characterize a feasible protocol of SWOP induction in mice.

Methods to precondition the myocardium include local ischemia, hypoxia, transient ischemia in a remote organ or pharmacologically [2-4, 8-14, 16-21]. Local ischemia is the oldest and most frequently used method to induce an early and late phase of preconditioning in mice. Guo et al. [16] described that a sequence of six cycles of 4 min coronary occlusion and $4 \mathrm{~min}$ reperfusion was protective in mice. With this technique the magnitude of protection afforded by the early phase was $75 \%$ reduction in infarct size and 48 $55 \%$ by the late phase of preconditioning. Similar, this protocol was highly effective in inducing SWOP in rabbits [2021].

The major disadvantage of regional cardiac ischemia as SWOP stimulus is the need to perform surgery to deliver it.
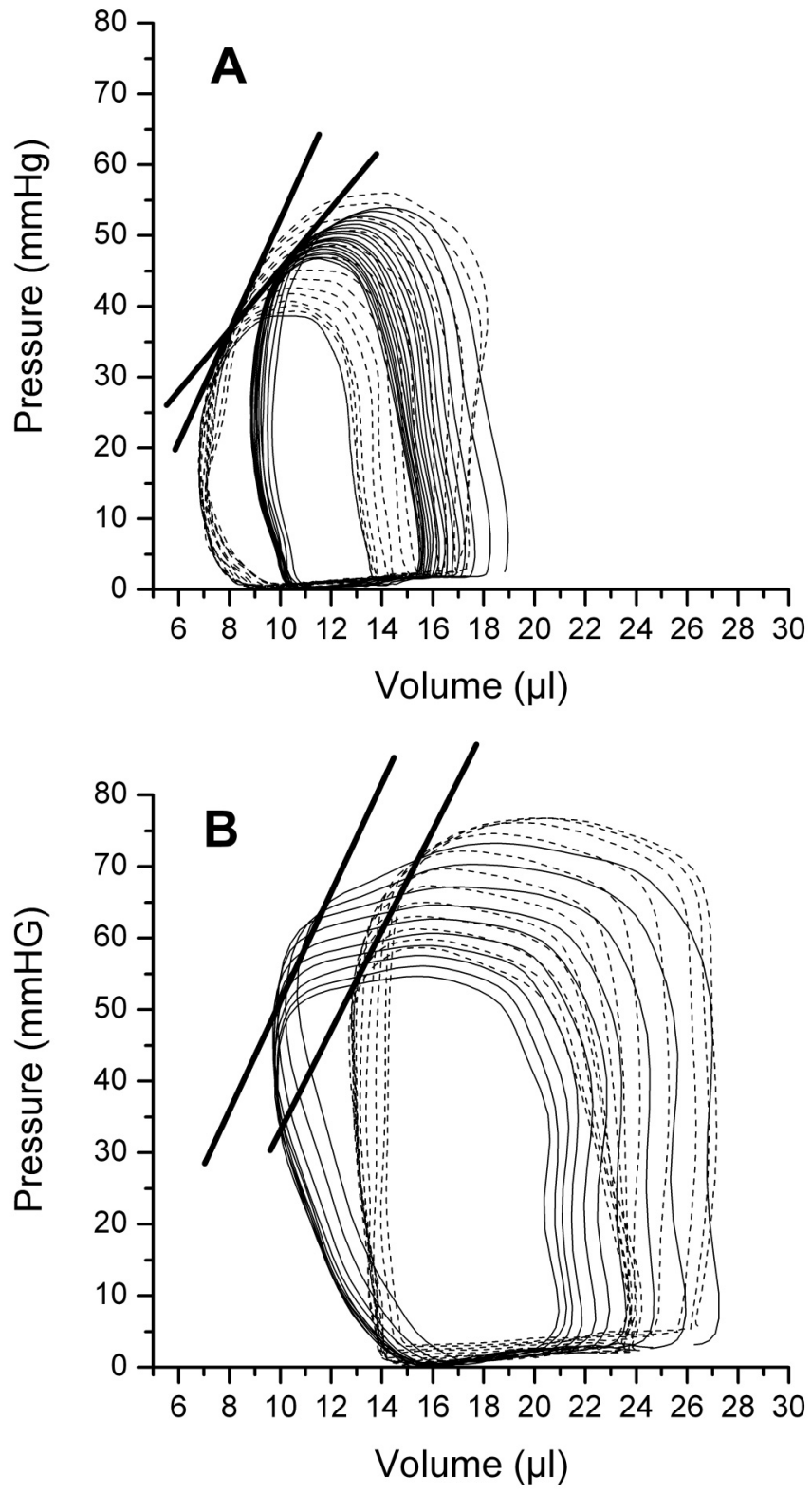

Fig. (1). (A) Representative example of occlusion loops and Eescurves of the ischemia groups. Group I + P: dash loops and Group I + NP: solid loops. (B) Representative example of occlusion loops and Ees-curves of the sham groups. Group sham + P: dash loops and Group sham + NP: solid loops.

The required survival period between the stimulus and the index ischemia, which often requires a second cardiovascular surgery in mice, often limits the applicability. Although this is a feasible technique in lean mice, we found in a preliminary study unacceptable mortality rates of more than $50 \%$ in the obese ob/ob mouse model. Not only the limited survival after surgery to induce regional cardiac ischemia is problematic, also the fact that anesthesia is necessary for it. This anesthesia can interfere in an uncontrolled way with the preconditioning stimulus, especially if volatile anesthetics or opioid agonists are used $[2,10,12]$.

Acute systemic and/or intermittent hypoxia was described in the early years 2000 as an effective preconditioning stimulus. Cai et al. [13] described for the first time the protocol of 5 cycles of 6 minutes, in which the oxygen con- 


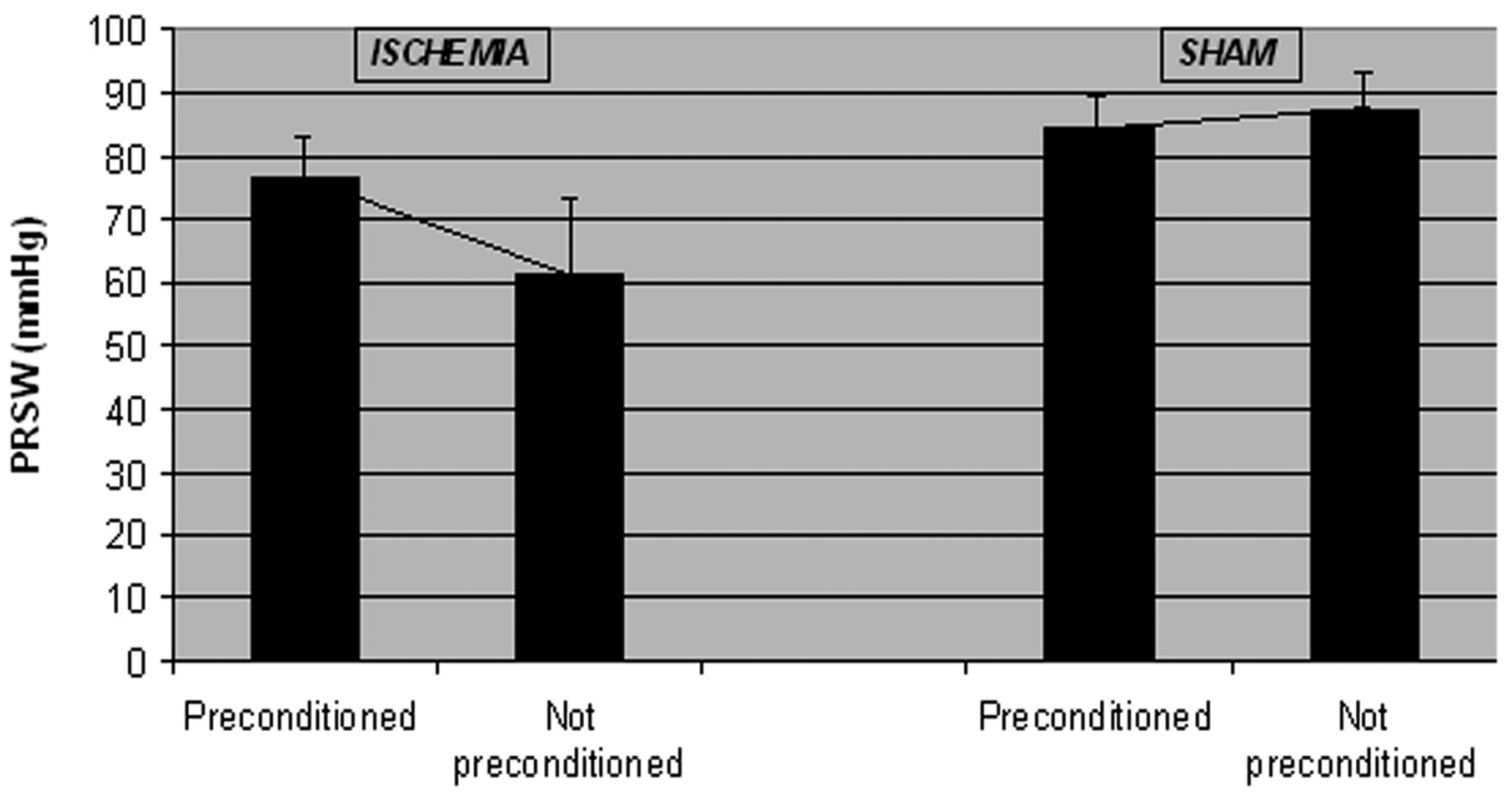

Fig. (2). PRSW is significantly $(\mathrm{p}=0.03)$ better preserved after the preconditioning protocol in the ischemia group. There were no statistical significant differences among the sham groups.

centration was rapidly reduced to $6 \%$. With this method he could induce myocardial protection in mice hearts studied 24 hours later in a Langendorff system. Ischemia was induced by a perfusion stop during 30 minutes. Left ventricular developed pressure improved and infarct size was reduced [13]. With this hypoxic preconditioning protocol, no right ventricular hypertrophy and pulmonary hypertension is seen [18]. Xi et al. [14] studied delayed preconditioning induced by one or two cycles of $10 \%$ hypoxia for various durations (30 min, $2 \mathrm{~h}, 4 \mathrm{~h}$ ). Infarct size was reduced only in mice pretreated with one or two cycles of 4 hour hypoxia.

To examine the in vivo effect of this stimulus, we performed cardiac pressure-conductance catheterization to determine left ventricular contractility with load-dependent and load-independent parameters [15, 22-24]. Infarct size measurements were used to identify the morphological damage after ischemia. In our series, there was no mortality with the described hypoxic preconditioning protocol.

Infarct size of the risk zone was significantly reduced from $49.9 \pm 7.3 \%$ to $24.7 \pm 7.3 \%$ after preconditioning. This reduction to about half the infarct size is comparable with the data of Guo et al. [16] who used local cardiac ischemia as preconditioning stimulus and the in vitro data of Cai et al. [13] with hypoxia as preconditioning stimulus.

Systolic function and active diastolic relaxation, as measured by ejection fraction and Tau were significantly better preserved. Ees and PRSW are considered as the gold standards to study ventricular contractility [24]. Accurate measurement of them requires the invasive procedure of pressure-volume measurements with different preload conditions. This parameters Ees and PRSW were respectively $42 \%$ and $25 \%$ better preserved by preconditioning after ischemia. This was never shown before.

The underlying mechanisms of the second window of preconditioning following hypoxic preconditioning in mice are not known. Cai et al. [13] showed that erythropoietin
(EPO) mRNA expression was induced in kidneys of wildtype mice subjected to intermittent hypoxia, resulting in increased plasma EPO levels. No other data are available concerning the second window of preconditioning after hypoxic preconditioning.

In rat models, the role of $\mathrm{NO}$ and mitochondrial $\mathrm{K}_{\mathrm{ATP}}$ channels in the hypoxic induced cardioprotection has been shown by Beguin et al. [25]. Rats, exposed to 4 hours of intermittent hypoxia had an infarct size reduction of $33.5 \%$ to $21.5 \%$. This effect was abolished by L-NAME, a NOS inhibitor and the $\mathrm{K}_{\mathrm{ATP}}$ channel blocker 5-HD. More recently, Beguin et al. [26] showed that delayed preconditioning in rats is mediated by protein kinase $\mathrm{C}$ and triggered by $\mathrm{p} 38$ MAPK and Erk1/2. Another mechanism for this protection in rats is the formation of reactive oxygen species. Kolar et al. [27] showed that the protective effect of chronic intermittent hypoxia in rats hearts was abolished by treatment with the antioxidant $\mathrm{N}$-acetylcysteine.

In mice models following an ischemic preconditioning protocol ( 6 cycles of $4 \mathrm{~min}$ occlusion $/ 4 \mathrm{~min}$ reperfusion), iNOS plays an important role. Guo et al. [28] showed that iNOS was increased with $40 \%$ in the ischemic/reperfused region. Our model provides a comparable degree of cardiac protection. It is thus tempting to suggest that iNOS is a potential mechanism in hypoxic preconditioning in mice.

Also COX-2 plays an important role. COX-2 activity was upregulated following ischemic preconditioning in mice, but, in contrast, the absence of iNOS prevented the activity of COX-2 protein. In the same study, it was found that iNOS and COX-2 coprecipitated 24 hours after ischemic preconditioning in the myocardium, indicating a physical interaction between these proteins. The upregulation of COX-2 protein expression after ischemic preconditioning was mediated by a JAK1/2-STAT1/3-signaling cascade [29].

In conclusion, this study shows that hypoxic preconditioning is a feasible stimulus to induce in vivo a second win- 
dow of preconditioning in mice. Cardiac contractility can significantly be preserved by this technique. The degree of cardiac protection induced by the intermittent hypoxia protocol seems to be comparable with regional cardiac ischemia as preconditioning stimulus.

\section{REFERENCES}

[1] Murry CE, Jennings RB, Reimer KA. Preconditioning with ischemia: a delay of lethal cell injury in ischemic myocardium. Circulation 1986; 74(5): 1124-36

[2] Bolli R. The late phase of preconditioning. Circ Res 2000; 87 (11): 972-83.

[3] Bolli R, Li QH, Tang XL, et al. The late phase of preconditioning and its natural clinical application - gene therapy. Heart Fail Rev 2007; 12 (3-4): 189-99.

[4] Baxter GF. Role of adenosine in delayed preconditioning of the myocardium. Cardiovasc Res 2002; 55 (3): 483-94.

[5] Gross GJ, Peart JN. Katp channels and myocardial preconditioning: an update. Am J Physiol 2003; 285 (3): H921-30.

[6] Hendrikx M, Toshima Y, Mubagwa K, Flameng W. Improved functional recovery after ischemic preconditioning in the globally ischemic rabbit heart is not mediated by adenosine A1 receptor activation. Basic Res Cardiol 1993; 88 (6): 576-93.

[7] Laude K, Beauchamp P, Thuillez C, Richard V. Endothelial protective effects of preconditioning. Cardiovasc Res 2002; 55 (3): 46673.

[8] Tanoué Y, Herijgers $\mathrm{P}$, Meuris $\mathrm{B}$, et al. Ischemic preconditioning reduces unloaded myocardial oxygen consumption in an in vivo sheep model. Cardiovasc Res 2002; 55 (3): 633-41.

[9] Valen G. Cellular signalling mechanisms in adaptation to ischemiainduced myocardial damage. Ann Med 2003; 35 (5): 300-7.

[10] Wakeno-Takahashi M, Otani H, Nakao S, Imamura H, Shingu K. Isoflurane induces second window of preconditioning through upregulation of inducible nitric oxide synthase in rat heart. Am J Physiol Heart Circ Physiol 2005; 289 (6): H2585-91.

[11] Xia Z, Herijgers P, Nishida T, et al. Remote preconditioning lessens the deterioration of pulmonary function after repeated coronary artery occlusion and reperfusion. Can J Anaesth 2003; 50 (5): 4818 .

[12] Yellon DM, Downey JM. Preconditioning the myocardium : from cellular physiology to clinical cardiology. Physiol Rev 2003; 83 (4): 1113-51.

[13] Cai Z, Manalo DJ, Wei G, et al. Hearts from rodents exposed to intermittent hypoxia or erythropoietin are protected against ischemia-reperfusion injury. Circulation 2003; 108 (1): 79-85.

[14] Xi L, Tekin D, Gursoy E, et al. Evidence that NOS2 acts as a trigger and mediator of late preconditioning induced by acute systemic hypoxia. Am J Physiol 2002; 283 (1): H5-H12.
[15] Van den Bergh A, Vanderper A, Vangheluwe P, et al. Dyslipidemia in type II diabetic mice does not aggravate contractile impairment but increases ventricular stiffness. Cardiovasc Res 2008; 77 (2): 371-9.

[16] Guo Y, Wu WJ, Qiu Y, et al. Demonstration of an early and a late phase of ischemic preconditioning in mice. Am J Physiol 1998; 275 (4Pt2): H1375-H1387.

[17] Kharbanda RK, Mortensen UM, White PA, et al. Transient limb ischemia reduces remote ischemic preconditioning in vivo. Circulation 2002; 106 (23): 2881-3.

[18] Ostadal B, Ostadalova I, Dhalla NS. Development of cardiac sensitivity to oxygen deficiency: comparative and ontogenetic aspects. Physiol Rev 1999; 79 (3): 635-659.

[19] Patel HH, Moore J, Hsu AK, Gross GJ. Cardioprotection at a distance: mesenteric artery occlusion protects the myocardium via an opioid sensitive mechanism. J Mol Cell Cardiol 2002; 34 (10): 1317-23.

[20] Qiu Y, Rizvi A, Tang XL, et al. Nitric oxide triggers late preconditioning against myocardial infarction in conscious rabbits. Am J Physiol 1997; 273 (6 Pt 2): H2931-H2936.

[21] Takano H, Manchikalapudi S, Tang XL, et al. Nitric oxide synthase is the mediator of late preconditioning against myocardial infarction in conscious rabbits. Circulation 1998; 98 (5): 441-449.

[22] Georgakopoulos D, Mitzner WA, Chen $\mathrm{CH}$, et al. In vivo murine left ventricular pressure-volume relations by miniaturized conductance micromanometry. Am J Physiol 1998; 274 (4 Pt 2): H141622.

[23] Van den Bergh A, Flameng W, Herijgers P. Type II diabetic mice exihibit contractile dysfunction but maintain cardiac output by favourable loading conditions. Eur J Heart Fail 2006; 8 (8): 777-83.

[24] Van den Bergh A, Flameng W, Herijgers P. Parameters of ventricular contractility in mice: influence of load and sensitivity to changes in inotropic state. Pflugers Arch 2008; 455 (6): 987-94.

[25] Beguin PC, Joyeux-Faure M, Godin-Ribuot D, Levy P, Ribuot C. Acute intermittent hypoxia improves rat myocardium tolerance to ischemia. J Appl Physiol 2005; 99 (3): 1064-1069.

[26] Beguin PC, Belaidi E, Godin-Ribuot D, Levy P, Ribuot C. Intermittent hypoxia-induced delayed cardioprotection is mediated by PKC and triggered by p38 MAP kinase and Erk 1/2. J Mol Cell Cardiol 2007 (2); 42: 343-51.

[27] Kolar F, Jezkova J, Balkova P, et al. Role of oxidative stress in PKC- $\delta$ upregulation and cardioprotection induced by chronic intermittent hypoxia. Am J Physiol 2007; 292 (1): H224-H230.

[28] Guo Y, Jones WK, Xuan YT, et al. The late phase of ischemic preconditioning is abrogated by targeted disruption of the inducible NO synthase gene. Proc Natl Acad Sci USA 1999; 96 (20): $11507-$ 11512.

[29] Xuan YT, Guo Y, Zhu Y, et al. Mechanism of cyclooxygenase-2 upregulation in late preconditioning. J Mol Cell Cardiol 2003; 35 (5): 525-537.

(C) Van der Mieren et al.; Licensee Bentham Open.

This is an open access article distributed under the terms of the Creative Commons Attribution License (http: //creativecommons.org/licenses/by/2.5/), which permits unrestrictive use, distribution, and reproduction in any medium, provided the original work is properly cited. 